\title{
Refractive Index of Uniaxially Stretched Poly(ethylene terephthalate) Film at Microwave Frequencies
}

\author{
Shigeyoshi OsaKI \\ Faculty of Education, Shimane University, Matsue, Shimane 690, Japan
}

(Received June 12, 1995)

\begin{abstract}
Refractive indices of uniaxially stretched poly(ethylene terephthalate) films with $9-10 \%$ crystallinity were determined at microwave frequencies. An empirical formula $n_{\mathrm{MW}}=0.0508+1.0681 n_{\mathrm{OPT}}$ was derived, with a correlation coefficient of 0.998 , where $n_{\mathrm{MW}}$ and $n_{\mathrm{OPT}}$ denote the refractive indices at $4.0 \mathrm{GHz}$ and $5.09 \times 10^{14} \mathrm{~Hz}$ ( $589 \mathrm{~nm}$ wavelength), respectively. The coefficient of 1.0681 between $n_{\mathrm{MW}}$ and $n_{\mathrm{OPT}}$ was smaller than that of 1.3561 in the previously determined empirical formula for biaxially stretched poly(ethylene terephthalate) films with $35 \%$ crystallinity. Such a difference in the coefficient may be ascribed to the difference in crystallinity between two kinds of films. It was found that $n_{\mathrm{MW}}$ decreased when the frequency increased from $4.0 \mathrm{GHz}$ to $12.2 \mathrm{GHz}$ but was distinctly higher than $n_{\mathrm{OPT}}$ and appeared to approach $n_{\mathrm{OPT}}$.

KEY WORDS Refractive Index / Birefringence / Poly(ethylene terephthalate) / Visible Ray / Microwave / Frequency Dependence /
\end{abstract}

The refractive index is widely used to estimate the optical anisotropy of polymer films. ${ }^{1}$ It can be measured relatively quickly, and a sample needed for it can be prepared relatively easily. However, this method can be of little use for opaque or colored films at visible wavelengths because of their poor transmission to visible rays. Furthermore, in the measurement at visible wavelengths, it is sometimes not easy to identify the boundary of a shade at the critical angle of total reflection for a film under the microscope.

Recently, we have developed a microwave method for determining the molecular orientation in polymer films. ${ }^{2-5}$ Thus, in one of our previous papers, we reported the refractive index at a microwave frequency of $4.0 \mathrm{GHz}$ on biaxially stretched films of poly(ethylene terephthalate) (PET). ${ }^{6}$ Since in the previous paper $^{6}$ our microwave method was applied only to biaxially stretched PET, however, it is uncertain whether the microwave method is simply applicable to uniaxially stretched PET.

The present paper describes the determination of the refractive index at microwave frequencies between 4.0 and $12.2 \mathrm{GHz}$ for uniaxially stretched PET films, the comparison of the refractive indices at $4.0 \mathrm{GHz}$ with those for biaxially stretched PET films, and looks at how the results can be correlated with the refractive index for visible rays.

\section{EXPERIMENTAL}

We chose for the present study two uniaxially stretched PET films with different thickness. Both had 9-10\% crystallinity at $20^{\circ} \mathrm{C}$ when estimated by the empirical formula between $\varepsilon^{\prime \prime}$ at $4.0 \mathrm{GHz}$ and crystallinity given by ref 3 . Here, $\varepsilon^{\prime \prime}$ denotes the dielectric loss. The size of the sample film was changed from $10 \mathrm{~cm} \times 10 \mathrm{~cm}$ to $3.5 \mathrm{~cm} \times 3.5 \mathrm{~cm}$ from 4.0 to $12.2 \mathrm{GHz}$ measurements. A given sample film was inserted between the narrow gap in the cavity resonator system consisting of a pair of rectangular waveguides and irradiated by a polarized microwave incident normally to it. The type of the waveguides was varied depending on the frequency. ${ }^{7,8}$ The effective area of the film for irradiation was a circle of $7.0 \mathrm{~cm}$ diameter at $4.0 \mathrm{GHz}$ and one of $2.5 \mathrm{~cm}$ diameter for $12.2 \mathrm{GHz}$. During the irradiation the film was rotated about the central axis normal to its plane to a series of angles, and at each angle its dielectric constant $\varepsilon^{\prime}$ was determined from the resonance frequency. Here, the resonance frequency refers to the frequency at the maximum transmitted microwave intensity in the resonance curve determined by our apparatus. ${ }^{3}$ Actually, $\varepsilon^{\prime}$ was calculatd from the equation:

$$
\varepsilon^{\prime}=1+A(c / t)\left(f_{1}-f_{2}\right) / f_{2}
$$

where $t$ is the thickness of the film, $c$ a parameter related to the depth of the waveguide, $A$ a constant associated with the instrument, and $f_{1}$ and $f_{2}$ the resonance frequencies with subscripts 1 and 2 referring to before and after the insertion of the film.

The refractive index $n$ for a sheet material can be derived from the Maxwell equation. ${ }^{9}$ When the dielectric loss of the material is sufficiently small and the magnetic permeability of the material may be assumed to be unity independently of the rotation angle $\theta, n$ as a function of $\theta$ can be related to $\varepsilon^{\prime}$ by

$$
n(\theta)^{2}=\varepsilon^{\prime}(\theta)
$$

Thus, we can evaluate $n(\theta)$ from the dielectric measurements at a series of $\theta$. Note that this evaluation can be made regardless of whether the sample is transparent or not for visible rays and that, at a fixed rotation angle, it took only $16 \mathrm{~s}$ with our apparatus. The measurement was made at five frequencies ranging from 4.0 to $12.2 \mathrm{GHz}$.

The refractive index for visible wavelength of $589 \mathrm{~nm}$ $\left(5.09 \times 10^{14} \mathrm{GHz}\right)$ was measured using an Abbe refractometer with the sample strips of $8 \mathrm{~mm} \times 20 \mathrm{~mm}$ cut out of the given PET film in different directions.

\section{RESULTS}

Figure 1 shows the angle dependence of $n_{\mathrm{MW}}$ at $4.0 \mathrm{GHz}$ 
and $n_{\mathrm{OPT}}$ for the uniaxially stretched PET film with $82 \mu \mathrm{m}$ thickness and $9 \%$ crystallinity. Here, $n_{\mathrm{MW}}$ and $n_{\mathrm{OPT}}$ denote the refractive indices at a microwave frequency and a visible light frequency, respectively. It is seen that $n_{\mathrm{MW}}$ is larger than $n_{\mathrm{OPT}}$ at any rotation angle. This difference may be mainly attributed to the different contributions of dielectric $\beta$-relaxation ascribed to the local motion in both amorphous and intercrystalline regions of the film.

Figure 2 shows the frequency dependence of $n_{\mathrm{MW}}$ in the machine direction MD (i.e., the direction of uniaxial stretching) and the transverse direction TD perpendicular to the MD for the uniaxially stretched PET film with $410 \mu \mathrm{m}$ thickness and $10 \%$ crystallinity. For either direction $n_{\mathrm{MW}}$ decreases with increasing frequency and appears to approach $n_{\text {OPT }}$. The distinct difference between $n_{\mathrm{MW}}$ and $n_{\mathrm{OPT}}$ indicates that at microwave frequencies the

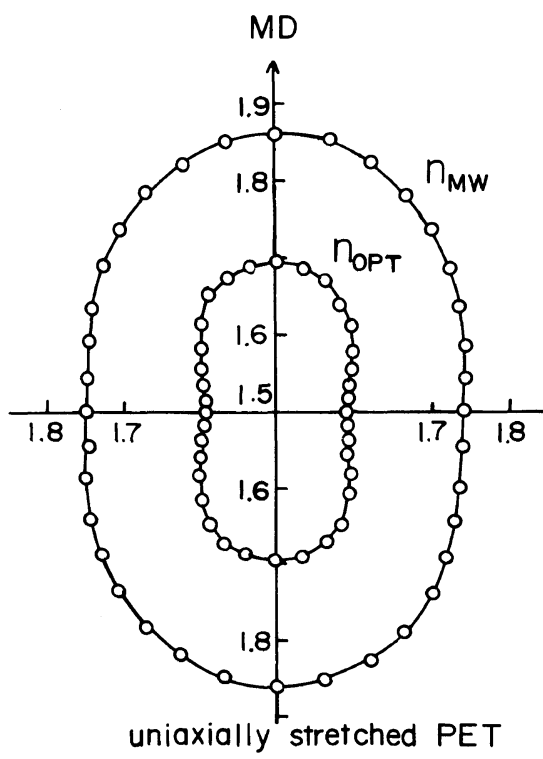

Figure 1. Angle dependence of refractive index at $4.0 \mathrm{GHz}\left(n_{\mathrm{MW}}\right)$ and $5.09 \times 10^{14} \mathrm{~Hz}\left(n_{\mathrm{OPT}}\right)$ for uniaxially stretched PET film with $82 \mu \mathrm{m}$ thickness and $9 \%$ crystallinity.

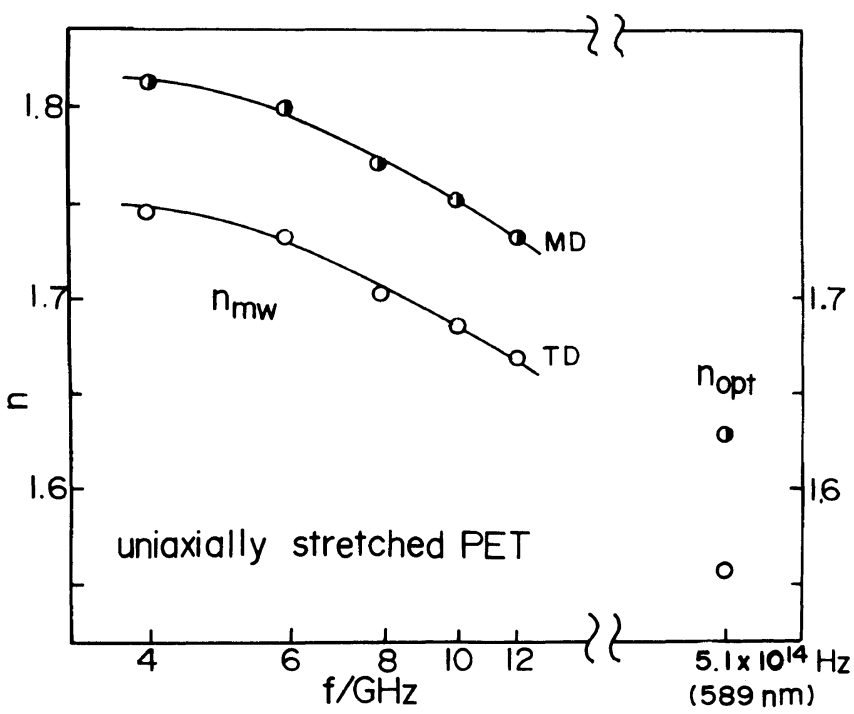

Figure 2. Frequency dependence of refractive index $n$ in the microwave region for uniaxially stretched PET film with $410 \mu \mathrm{m}$ thickness and $10 \%$ crystallinity. Half-filled circles, data in the MD (direction of uniaxial stretching); unfilled circles, data in the TD, perpendicular to the MD. For comparison the corresponding refractive index values at $5.09 \times 10^{14} \mathrm{~Hz}$ (589 nm wavelength) are included. refractive index has contributions from the dielectric $\beta$-relaxations relating to the local motions as well as the polarization due to the distortion of the PET molecules. The refractive index in the MD was consistently higher than that in the TD for the uniaxially stretched PET films studied here. Furthermore, the maximum refractive index appeared in the MD; this is expected because the PET chains are forced to orient mainly in the MD.

From the data of Figure 1 we can read a pair of $n_{\mathrm{MW}}$ and $n_{\mathrm{OPT}}$ values at any fixed rotation angle. The half-filled circles in Figure 3 show plots of $n_{\mathrm{MW}}$ against $n_{\mathrm{OPT}}$ made from such data for different rotation angles. They give a linear relation

$$
n_{\mathrm{MW}}(4.0 \mathrm{GHz})=0.0508+1.0681 n_{\mathrm{OPT}}
$$

with a correlation coefficient of 0.998 . This finding indicates that whether a given film is transparent or not, its refractive index for visible light can be estimated from the dielectric constant measurement at a microwave frequency. For comparison, our previous data for a biaxially stretched PET film with $35 \%$ crystallinity are quoted with unfilled circles in Figure 3. They also fall on a straight line, but with a slope $1.36-1.38$. It is seen that when compared at the same $n_{\mathrm{MW}}, n_{\mathrm{OPT}}$ for uniaxially stretched film is smaller than that for the biaxially stretched one. Equivalently, at the same $n_{\mathrm{OPT}}, n_{\mathrm{MW}}$ for the former is consistently higher than that for the latter.

We denote $\Delta n$ as the difference between $\mathrm{n}$ in a given direction (rotation angle) and that in the direction perpendicular to it. This quantity may be taken as a convenient measure of film's anisotropy. By varying the given direction we can see from the data of Figure 1 how $\Delta n_{\mathrm{MW}}$ and $\Delta n_{\mathrm{OPT}}$ change with $\theta$. In Figure $4, \Delta n_{\mathrm{MW}}$ at $4.0 \mathrm{GHz}$ is plotted against the corresponding $\Delta n_{\mathrm{OPT}}$. The

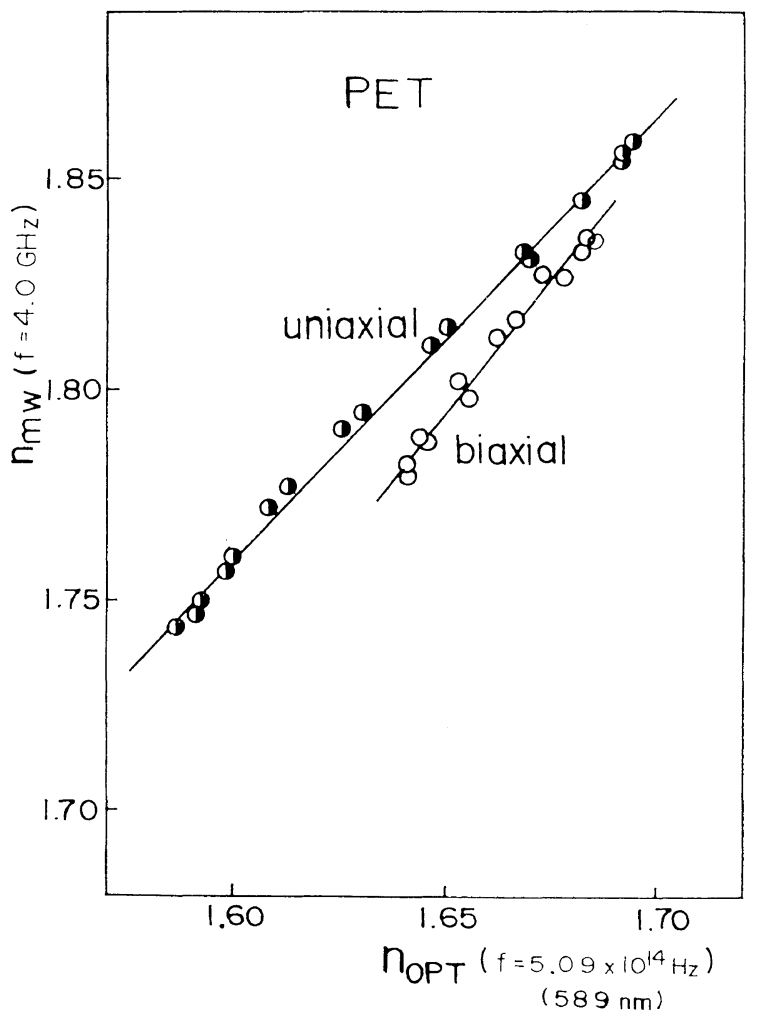

Figure 3. $n_{\mathrm{MW}}$ at $4.0 \mathrm{GHz}$ plotted against $n_{\mathrm{OPT}}$ for uniaxially stretched PET film with $9 \%$ crystallinity (half-filled circles) and for biaxially stretched PET film with $35 \%$ crystallinity (unfilled circles). 


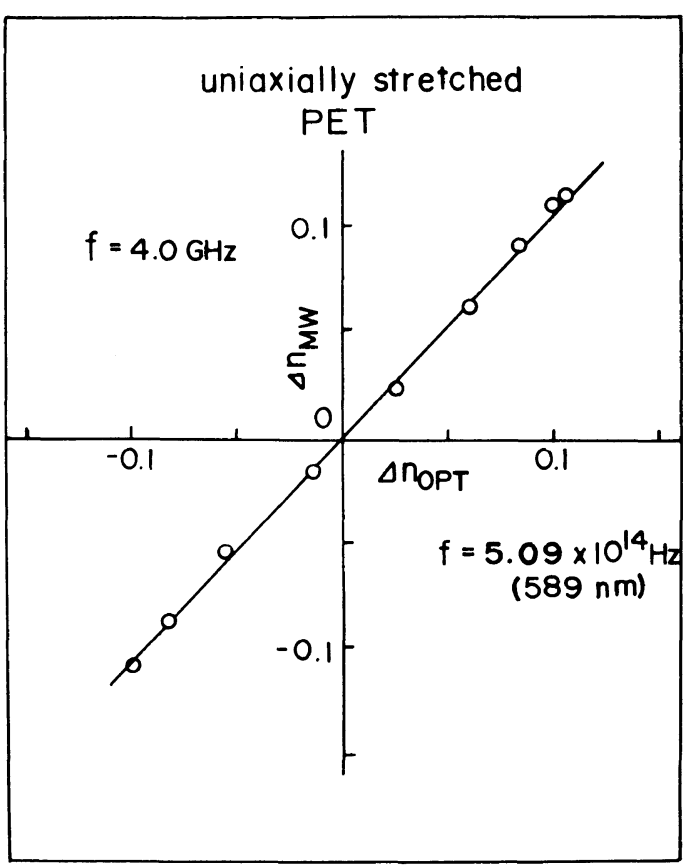

Figure 4. $\Delta n_{\mathrm{MW}}$ at $4.0 \mathrm{GHz}$ plotted against $\Delta n_{\mathrm{OPT}}$ for uniaxially stretched PET film with $82 \mu \mathrm{m}$ thickness.

data points are fitted by

$$
\Delta n_{\mathrm{MW}}=0.0017+1.0626 \Delta n_{\mathrm{OPT}}
$$

with a correlation coefficient of 0.999 . This indicates that the difference between $\Delta n_{\mathrm{MW}}$ at $4.0 \mathrm{GHz}$ and $\Delta n_{\mathrm{OPT}}$ is relatively small for our uniaxially stretched PET, as compared with the result for biaxially stretched PET. ${ }^{6}$

\section{DISCUSSION}

The behavior shown in Figure 2 that either $n_{\mathrm{MW}}$ for the MD or the TD decreases with increasing frequency may be considered reflecting that the refractive index of a polymer film in the microwave region is governed first by the dielectric $\beta$-relaxation due to the local motion of the chain and then by the polarization due to the distortion of the molecular chain ${ }^{10}$ as the frequency is increased. It is mainly the electronic polarization due to the displacement of electron clouds around the polymer molecules that contributes to $n_{\mathrm{OPT}}$.

The slopes of the lines drawn in Figure 3 are 1.068 and 1.356 for the uniaxially stretched PET and the biaxially stretched one, respectively. This difference is distinct, and may be attributed to the big difference in crystallinity between the two.

The coefficients in the empirically found relations (3) and (4) may vary with the degree of stretching, crystallinity, and so on of given PET samples, so that they may not be generally applied for evaluating $n_{\mathrm{OPT}}$ and $\Delta n_{\mathrm{OPT}}$ from dielectric measurements of $n_{\mathrm{MW}}$ and $\Delta n_{\mathrm{MW}}$. However, the linear correlations between $n_{\mathrm{MW}}$ and $n_{\mathrm{OPT}}$ and between $\Delta n_{\mathrm{MW}}$ and $\Delta n_{\mathrm{OPT}}$ are believed to hold in general for PET. Thus, our microwave apparatus, which allows $n_{\mathrm{MW}}$ and $\Delta n_{\mathrm{Mw}}$ to be determined quickly, should be useful when information on the optical anisotropy of PET film for visible rays is desired. This is especially true when the film is opaque or colored. The dielectric determination of refractive index is somewhat less accurate than the Abbe refractometer method used at visible light wavelengths, but this is not a series problem for most practical purposes.

Here, it should be noted that the circular area of a sample film effective for microwave irradiation had $60 \mathrm{~mm}$ diameter for measurements of $n_{\mathrm{MW}}$ at $4.0 \mathrm{GHz}$. Uniaxially stretched PET films are generally affected by edge effects due to the machine conditions for producing films, different from biaxially stretched PET films. Such edge effects sometimes give the non-uniformity of orientation within the large circular area of the uniaxially stretched PET sample. Thus, such a difference in production method may also give the difference in empirical equations between uniaxially and biaxially stretched PET films. In this study, however, we supposed that the refractive index at $n_{\mathrm{Mw}}$ should be uniform within the effective sample circle since samples were prepared from the middle part of the uniaxially stretched PET film.

In the near future a general equation, which is applicable to both the uniaxially and biaxially stretched PET films, will be presented.

\section{REFERENCES}

1. I. M. Ward, "Structure and Properties of Oriented Polymers," Applied Science, London, 1975.

2. S. Osaki, Polym. J., 19, 821 (1987).

3. S. Osaki, J. Appl. Phys., 64, 4181 (1988).

4. S. Osaki, J. Appl. Phys., 67, 6513 (1990).

5. S. Osaki, Nature, 347, 132 (1990).

6. S. Osaki and K. Uranishi, Polymer, 31, 33 (1990).

7. S. Osaki, J. Polym. Sci., Part C, 28, 147 (1990).

8. S. Osaki, J. Polym. Sci., Phys. Ed., 33, 685 (1995).

9. H. M. Altschler, in "Handbook of Microwave Measurements," M. Suche and J. Fox, Ed., Wiley, New York, N.Y., (1963).

10. W. Meyer, "Proceedings of the VIth International Cryogenic Engineering Conference," K. Mendelssohn, Ed., IPC Science and Technology Press, Guildford, 1976. 